\title{
Hepatitis B immunisation of household contacts: retrospective study of vaccine coverage
}

\author{
G Richardson, M R Evans, D Westmoreland
}

The United Kingdom has a policy of selective hepatitis B immunisation of people at high risk of infection including sexual or close household contacts of a case or carrier of hepatitis B, and babies born to infected mothers. ${ }^{12}$ Risk of progression to chronic hepatitis B disease is greatest among infants infected at birth (90\%), and children infected between ages $1-5$ years (25-50\%). ${ }^{3}$ We investigated whether the UK policy is being effectively implemented.

\section{Methods and results}

Hepatitis B carriers resident in Bro Taf Health Authority were identified from virology laboratory records for 1993 to 1995 . We excluded blood donors, occupational health or genitourinary medicine (anonymous) referrals and patients who had died. Carriers were classified as high risk ( $\mathrm{HBeAg}$ positive and anti-HBe negative), intermediate risk (negative for " $e$ " markers) or low risk (HBeAg negative and anti-HBe positive). Information about household contacts was obtained by sending a questionnaire to the index patient's primary care doctor who was also asked what advice they had received on follow up of household contacts and about their knowledge of immunisation recommendations. Non-responders were followed up by telephone.

We identified 125 carriers and excluded 34. Questionnaires were returned for 62 of 91 eligible patients, a $68 \%$ response rate. Of these, 17 were no longer registered with the practice and were untraceable through the family health register, one had duplicate registration and one questionnaire was returned unanswered, giving data on 43 patients. Median age of patients was 35 years (range 18-78), 29 (67\%) were female and $31(72 \%)$ were from ethnic minorities. There were $8(19 \%)$ high risk, $3(7 \%)$ intermediate risk and $32(74 \%)$ low risk carriers. Antenatal screening had identified 21 patients (49\%).

Public Health

Directorate, Bro Taf

Health Authority, Cathays Park, Cardiff CF10 3NW, UK G Richardson

M R Evans

Cardiff Public Health

Laboratory, University Hospital of Wales, Cardiff

D Westmoreland

Correspondence to:

Dr Richardson

(gill.richardson@)

bro-taf-ha.wales.nhs.uk)

Accepted for publication 6 June 2001 tacts (see table 1). Median age of contacts 13 years (range $1-75), 41(45 \%)$ were female and $82(90 \%)$ were from ethnic minorities. Contacts included $18(20 \%)$ sexual partners and $51(56 \%)$ children. Only 25 (27\%) contacts were fully immunised including 3 of $18(17 \%)$ sexual partners, and 6 of $31(19 \%)$ other household contacts, all children. Of infants born to infected mothers 15 of 42 $(36 \%)$ had received no immunisations and only 16 of $42(38 \%)$ were fully immunised.

Contacts of high risk cases were no more likely to be fully immunised than contacts of low risk cases ( 7 of 17 versus 18 of $74, p=0.27$ ). Antibody levels were checked after immunisation in only 3 of $25(12 \%)$ fully immunised contacts.

Primary care doctors had not been informed of the diagnosis of seven patients. Forty one (95\%) doctors were aware that sexual partners should be immunised but only 29 (67\%) knew that immunisation was recommended for other household contacts. Doctors received advice about immunisation of contacts for only 15 $(35 \%)$ patients. This was no more likely for high or intermediate risk than for low risk patients ( 4 of 11 versus 11 of $32, p=0.80$ ). However, contacts were more likely to be immunised ( $\geqslant 1$ dose) if the doctor had been given advice (24 of 42 versus 11 of 48 , $\mathrm{p}=0.0002$ ).

\section{Comment}

The UK's selective hepatitis B immunisation policy is failing many of those most at risk. Fewer than half of babies of carrier mothers and only a quarter of household contacts were fully immunised. Several measures could be taken to increase coverage including improving communications between the diagnosing laboratory, maternity unit and primary care, as well as between health professionals and patients. Ethnic minority patients may be disadvantaged because of insufficient information in languages other than English.

Babies of carrier mothers and sexual contacts of acute cases are priority groups. The UK has recently introduced universal antenatal screening, yet the challenge of comprehensive immunisation remains. Although selective immunisation is possibly a more cost efficient option, unless the current strategy can be fully implemented, universal hepatitis B immunisation may be the only effective strategy in the long run. ${ }^{45}$

\section{Contributor}

MRE initiated the study. GR, MRE and DW developed the original idea, designed the project and contributed to writing the paper. GR collected and analysed the data and is guarantor for the paper.

Table 1 Number of hepatitis B vaccine doses per contact according to infectivity of the index patient

\begin{tabular}{lllllll}
\hline \multirow{2}{*}{$\begin{array}{l}\text { Hepatitis B status of } \\
\text { patient }\end{array}$} & & \multicolumn{4}{l}{ Number (\%) of contacts immunised } \\
\cline { 6 - 7 } & Patients (n) & Contacts (n) & No doses & 1 dose & 2 doses & $\geqslant 3$ doses \\
\hline High risk & 8 & 14 & $9(64)$ & $1(7)$ & $0(0)$ & $4(29)$ \\
Intermediate risk & 3 & 3 & $0(0)$ & $0(0)$ & $0(0)$ & $3(100)$ \\
Low risk & 32 & 74 & $47(64)$ & $3(4)$ & $6(8)$ & $18(24)$ \\
Total & 43 & 91 & $56(62)$ & $4(4)$ & $6(7)$ & $25(27)$ \\
\hline
\end{tabular}


Funding: none.

Conflict of interests: none.

1 United Kingdom Health Departments. Immunisation against infectious disease. London: HMSO, 1996.

2 NHS Executive. Screening of pregnant women for hepatitis $B$ and immunisation of babies at risk. Health service circular. HSC 1998/127.
3 Benenson AS, ed. Control of communicable disease manual. Washington: American Public Health Association, 1995. Washington: American Public Health Association, 1995. tis B immunisation strategy - where now? Commun Dis tis B immunisation strategy

Public Health 1998;1:79-83.
Edmunds WJ. Universal or selective immunisation against hepatitis $\mathrm{B}$ virus in the United Kingdom? A review of recent cost-effectiveness studies. Commun Dis Public Health 1998;1:221-8

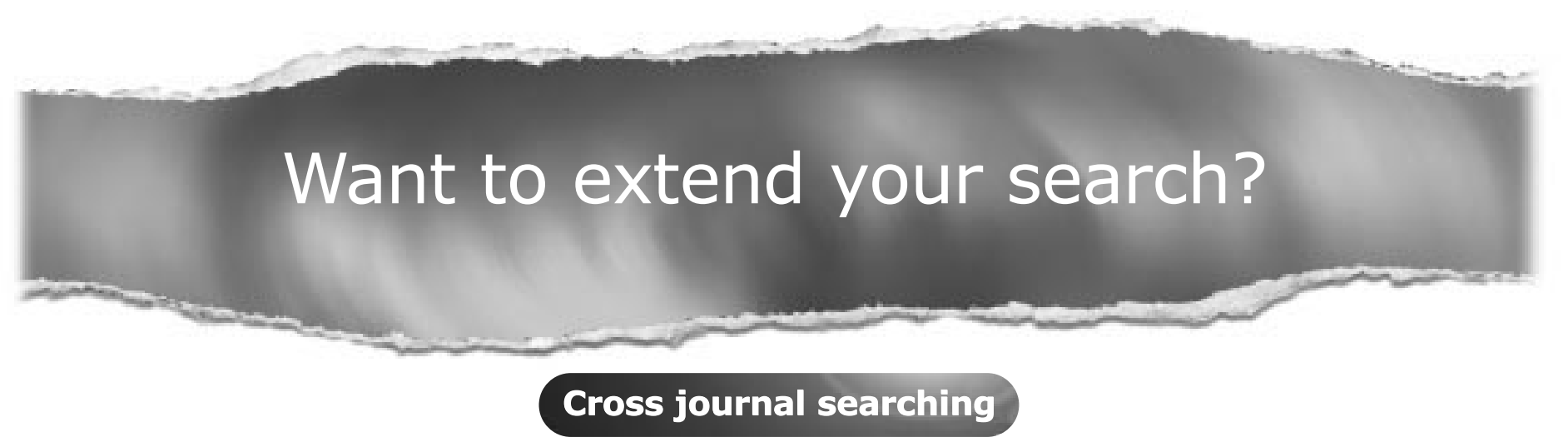

If you can't find what you are looking for in the Journal of Epidemiology and Community Health you can extend your search across many of the more than 200 journals available for selection. You can restrict your search to specific subject areas (eg, clinical medicine, basic research), or select specific journals, or search all available titles.

www.jech.com 